\title{
Cádiz: Plataforma de emigración a Indias. 1750-1760
}

\section{GRUPO INDIANO (*)}

\section{INTRODUCCION}

Desde que Magnus Mörner (1) publicara su conocido artículo sobre el estado de los conocimientos historiográficos de la emigración española al Nuevo Mundo no se ha vuelto a publicar un trabajo que refleje a modo de síntesis las aportaciones posteriores y el avance conseguido en semejante campo de la investigación histórica. Sin embargo, a partir de entonces se completaron los Catálogos de Pasajeros para todo el siglo XVI (2), se han multiplicado trabajos y estudios que contemplan la emigración desde el ámbito local, comarcal y en algunos casos regional y se han publicado las Actas de la 1..$^{\underline{a}}$ Reunión Científica de la Asociación de Historia Moderna, cuya segunda sección se desarrolló bajo el título La emigración española a Ultramar, 14921914, coordinada por Antonio Eiras Roel y las del II Congreso de la Asociación de Demografía Histórica (3).

A pesar de ello es mucho lo que aún queda por saber atribuible sobre todo a las dificultades que plantea el tema, tanto de orden metodológico -problemas que derivan de las fuentes disponibles-, como

(*) Este trabajo ha sido realizado por: Ana M. Fernández Zampaña, Encarnación de la Pascua Sánchez, M. ${ }^{a}$ Isabel Marmolejo López, J. Manuel de la Pascua Sánchez, Isabel Ramírez Rodríguez y M. ${ }^{a}$ de los Angeles Vicenti Alconchel, integrantes del grupo de investigación "Indiano", que ha contado para el presente proyecto con subvención de la Universidad de Cádiz (Programa de Grupos Precompetitivos).

(1) MORNER, Magnus: "La emigración española al Nuevo Mundo antes de 1810. Un informe del estado de la investigación", Anuario de Estudios Americanos, XXXII, 1975.

(2) Catálogo de Pasajeros; Vol. I, 1509-1534; Vol. II, 1535-1538; Vol. III, 1539-1559, Sevilla, 1940-1946. Vol. IV, 1560-1566; Vol. V, Tomo I, 1567-1574; Vol. V, Tomo II, 1575-1577, Madrid, 1980. Vol. VI, 1578-1585; Vol. VII, 1586-1599, Madrid, 1986.

(3) Là emigración española a Ultramar, 1492-1914. Actas de la I Reunión Científica de la Asociación de Historia Moderna, Madrid, 1991, y Emigración española y portuguesa a América. Actas del II Congreso de la Asociación de Demografia Histórica. Alicante, 1990 , vol. I. 
de interpretación de los datos conseguidos. Pero si la dificultad es mucha para los siglos XVI y XVIII, ésta se acrecienta para el siglo XVIII. En efecto, como apunta el profesor Eiras Roel (4), el siglo XVIII representa en este sentido un enigma; y lo es tanto por la inexistencia de datos empíricos suficientes como por el problema añadido de la representatividad de los registros oficiales teniendo en cuenta la importancia creciente, o constante, del fraude (5).

Estudios recientes permiten matizar afirmaciones tales como la disminución progresiva del número de emigrantes españoles a Indias a partir del siglo XVIII, si bien parece confirmarse la disminución de los efectivos andaluces (6) y del centro peninsular, en beneficio de los procedentes de las provincias norteñas y el aumento de la presencia catalana en América a partir del segundo tercio del siglo XVIII (7). Queda no obstante un amplio campo para la investigación pues son muchas las preguntas que quedan sin respuestas sobre todo para el siglo que nos ocupa (8). En efecto, el volumen total de la emigración, si esta es

(4) EIRAS ROEL, A.: "Presentación del volumen" La emigración española a Ultramar, op. cit., pp. 12-13.

(5) La emigración ilegal es difícil de documentarse "per se", no obstante la magnitud del fenómeno puede medirse con alguna extensión por el número de pasajeros libres (polizones) aprehendidos por las autoridades, MORNER, Magnus: op. cit., p. 8. Señalaremos también que la mayor libertad de navegación y comercio en el siglo XVIII, iniciada con la introducción del sistema de registros sueltos en 1739, y aumentada con los decretos de libre comercio, multiplica las dificultades para cuantificar el flujo migratorio partiendo de fuentes oficiales, precisamente en la época en que debió de crecer más. VAZQUEZ DE PRADA, V. y AMORES CARREDANO, J.B.: "la emigración de navarros y vascongados al N. Mundo y su repercusión en las comunidades de Origen" La emigración... op. cit., p. 135. Evidencia, asimismo, de este fenómeno son las disposiciones legales encaminadas a atajar este fraude que fueron numerosas a lo largo del XVIII, y en el mismo texto de muchas de ellas se hace constar explícitamente el volumen que habian alcanzado estas transgresiones, como por ejemplo en la de 1739 , donde se habla del descubrimiento de 170 polizones en las galeras que llegaron a tierra firme en 1737. GARCIA-BAQUERO, A. Cádiz y el Atlántico (1717-1778), Sevilla, 1976.

(6) La emigración andaluza en el siglo XVII se concentra en las tres primeras décadas del siglo (73,20\% del total del siglo). DIAZ-TRECHUELO, Lourdes: "La emigración familiar andaluza a América" en La emigración española... op. cit., pp. 189-197.

(7) EIRAS ROEL, A.: "Presentación del volumen" La emigración española... op. cit., pp. 12-13.

(8) En efecto para el siglo XVI contamos con los Catálogos de Pasajeros, con los trabajos de R. Konetzke, Magnus Morner, Boyd-Bowman, Pieter Jacobson; el siglo XVII, al menos para Andalucía cuenta con el importante trabajo realizado por investigadores de la Universidad de Córdoba bajo đirección de Lourdes Díaz-Trechuelo. Sin embargo, el siglo XVIII ha sido muy escasamente investigado contando tan sólo con trabajos dispersos y puntuales que no sobrepasan el marco local. 
familiar o se trata de individuos desclasados que emprenden la aventura de cruzar el océano en solitario, los ritmos de esta emigración, con sus momentos álgidos y sus declives, estableciendo una posible causalidad con acontecimientos en la península o en América (9), la procedencia de los emigrados y sus puntos de destino, sus conexiones con sus familiares y amigos que quedaron en la metrópoli, el perfil humano y social de estos emigrantes, etc., constituyen un amplio abanico de incógnitas que los investigadores actuales deberíamos resolver.

Nuestra investigación se ha limitado a la década central del siglo XVIII, a los años comprendidos entre 1750 y 1760 utilizando como fuente principal los fondos del Archivo Histórico Provincial de Cádiz (sección Protocolos); asimismo, y a fin de que sus datos sirvieran de contraste con los anteriores hemos utilizado los fondos del Archivo General de Indias, sección Ultramar. La importancia de los protocolos notariales para el estudio de la emigración, tanto interior como exterior, ha sido puesta de manifiesto recientemente y ya son muchos los trabajos publicados que han utilizado esta documentación como base fundamental (10). En efecto, dada la total ausencia de fuentes estadísticas precisas para toda la Edad Moderna y la parcialidad de los datos reflejados por la única fuente sistemática de que disponemos, a saber, las licencias de embarque, que nos hablan tan sólo de una parte de esta emigración, la emigración legal, los fondos notariales aparecen como necesarios e incluso insustituibles a pesar de la laboriosidad que entraña su consulta y su difícil exhaustividad.

\section{FUENTES}

La necesidad de clasificar la documentación en función de las personas que marchaban y no de las que otorgaban los documentos nos condujo a realizar un listado alfabético de emigrantes, en él incluimos todos los datos que hacían referencia a cada individuo. Lógicamente el número de personas es inferior al número total de documentos que ascienden a un total de 2.784 repartidos entre varios tipos documentales:

(9) Entre los factores de empuje o "expulsión" aparece como determinante la presión demográfica, MORNER, M.: op. cit., p. 31. EIRAS ROEL: op. cit., p. 13.

(10) Ya Richard Konetzke había indicado la importancia del Archivo de Protocolos Notariales de Sevilla en "Fuentes para la Historia demográfica de Hispanoamérica durante la época colonial", Anuario de Estudios Americanos, V, 1948, p. 6. Posteriormente la necesidad de salvar las lagunạs que ofrecen los Catálogos derivadas de su condición de fuente oficial hicieron proliferar los trabajos basados en otras fuentes alternativas como son los protocolos notariales. 
-Testamentos: 368 que suponen el 13,2\% de la documentación. -Licencias: 220 que suponen el 8\% de la documentación.

-Prorrogaciones: 40 que suponen el 1,4\% de la documentación. -Poderes: 2.156 que suponen el 77\% de la documentación.

Tal cantidad de documentos es el fruto de un barrido total de todas las notarías gaditanas en este período -25 notatías en años máximos que generan un total de 28 a 30 protocolos anuales-. Era necesaria, pues, una acción selectiva, y por ello nos centramos en la tipología más representativa para nuestros fines, aquella que nos diera la máxima información personal posible sobre emigrados y familiares. La mayoría de los documentos contenidos en nuestro muestreo son otorgados por hombres, a pesar de lo cual no debemos infravalorar la presencia de la mujer, puesto que domina en dos tipos documentales concretos: licencias y prorrogaciones; en ambos el papel de la mujer es fundamental.

El testamento es un tipo documental de una riqueza extraordinaria, nos informa sobre la naturaleza del testador, su estado civil, fecha y lugar del matrimonio, nombre del cónyuge, número de hijos, bienes, deudas y motivo por el que testa, dato éste muy importante para nosotros pues nos permite seleccionar aquellos que guardan relación con un futuro viaje a las Indias. Por su parte, el testamento redactado por una mujer nos habla tanto de un futuro viaje como de la ausencia del marido, siendo mucho más escaso, $16 \%$, que el redactado por hombres, $76,3 \%$.

Las Licencias poseen una tipología muy variada, en general se trata de la concesión de un permiso de una persona a otra para realizar una determinada actividad. Puede ser un permiso otorgado por un hombre para que su esposa haga un viaje, se beneficie de una herencia u otorgue algún tipo de documento. Especial mención merecen los otorgados por la mujer, puesto que generalmente se trata de una licencia concedida a su marido para la realización de un viaje. La información novedosa que nos aporta este tipo documental es su referencia al tiempo aproximado de permanencia en el Nuevo Mundo que va, desde la fórmula ambigua e indeterminada "todo el tiempo que necesitare", a los casos en donde se concretan el mes e incluso el día en que finaliza la vigencia del documento. Aquellas licencias que no mencionan el tiempo que permanece en vigor el documento representan el $40,4 \%$ del total, mientras aquellas que hacen una referencia temporal precisa quedan subdivididas de la siguiente manera: 
1. Aquellas que hacen estricta referencia al tiempo de duración del viaje bajo la fórmula "por la duración del viaje". Generalmente suele ser de un año y en la mayoría de los casos son concedidas a los tripulantes de los distintos navíos que emprendían la marcha. Representan el 3\% del total.

2. Aquellas licencias concedidas por un tiempo de tres años. Se trata del grupo más numeroso, $29,5 \%$ del total y junto con el grupo de menos de tres años es el más utilizado por los comerciantes.

3. Aquellas que se conceden por más de tres años, oscilando entre los cuatro y los nueve años. Generalmente son las licencias utilizadas por las personas que marchan al Nuevo Mundo para desempeñar un cargo público. Representan el 23,1\% del total.

4. Aquellas que se conceden por un tiempo inferior a los tres años. Como mencionamos anteriormente son utilizadas básicamente por los comerciantes y representan el $4 \%$ del total.

Las Prorrogaciones se otorgan con objeto de ampliar el tiempo de estancia del emigrado en su lugar de destino. Generalmente la mujer alega que su esposo no ha podido concluir las dependencias que llevaba a su cargo, siendo por tanto necesario una ampliación del tiempo de permiso de estancia fuera del hogar. En este caso predominan aquellas cuya duración es inferior a tres años con un 35\% del total. No eran extrañas las ausencias prolongadas si bien, lo más común era la pérdida de noticias sobre el emigrado de tal forma que aquellos que seguían en contacto con su familia y solicitaban las prorrogaciones fueron minoría. Y ello a pesar de que la falta de ambos documentos en una persona casada suponía la ilegalidad de la marcha y de su estancia en América. En este sentido hemos constatado incluso personas que cambiaban de identidad para pasar desapercibidas y no ser localizadas por su mujer, iniciando de esta forma una nueva vida.

El 100\% de las prorrogaciones fueron otorgadas por mujeres, mientras que las licencias lo fueron en un 99,09\%, pues en dos casos fueron otorgadas por hombres. El primero de ellos es un señor que concede a su esclavo un permiso para viajar a Indias (11), el segundo nos habla de un matrimonio, ella criada del marqués de las Amarillas que parte al Nuevo Mundo para desempeñar un cargo público, en este caso la licencia es otorgada por el marido para que su esposa pueda seguir trabajando para el marqués todo el tiempo que fuese necesario (12).

(11) Archivo Histórico Provincial de Cádiz (A.H.P.C.), sección protocolos, legajo 5.745, pp. 105-106.

(12) A.H.P.C., secc. protocolos', legajo 3.839, pp. 58-59. 
La emigración generaba un alto índice de documentos si bien no siempre encontramos los testamentos y licencias que verifiquen la partida de una persona, en estos casos recurrimos a los Poderes, a través de los cuales obtenemos como mínimo, la identidad del emigrante y su destino, no siendo infrecuente encontrarnos con la notificación de su naturaleza, vecindad, profesión, estado civil, nombre de la esposa... Su tipología es muy variada tanta como los objetivos y fines para los que se otorgan aunque no es difícil obtener una clasificación de los más frecuentes. Los poderes para testar son los que, a falta del testamento, nos ofrecen una información más rica, siendo a su vez un tipo documental muy frecuente, el 22,9\% del total. Los poderes para cobrar son los más numerosos, $41,8 \%$ del total, siendo diversas las causas de su expedición, desde cobrar soldadas hasta cobrar deudas, herencias... Poderes para administrar las haciendas, negocios, etc. 3,8\%. Poderes generales, denominado así el documento que autorizaba a una o varias personas la realización de determinadas actividades y que suponen el $18,5 \%$ del total. Los poderes para buscar marido pese a su rareza, sólo representan el $0,7 \%$ del total de la muestra, son interesantes pues informan de manera directa de la pérdida de contacto del emigrado con su familia. Por último y representando un 12,9\% del total, nos encontramos con poderes otorgados por los más variados motivos, para vender, casarse, bautizar a un hijo, etc.

Las licencias de embarque, obtenidas en el Archivo General de Indias, nos sirvieron para contrastar los datos obtenidos en las fuentes notariales. La década estudiada nos arroja un total de 3.470 personas, distribuidas en tres grupos: Provistos, Cargadores y Pasajeros. Son denominados provistos las personas que hacen el viaje para ejercer un cargo público otorgándoseles las licencias para ellos, su familia y servidumbre. Los cargadores son los miembros de la tripulación que, en su mayoría, portaban un cajón de mercancías. Dentro del grupo de pasajeros tienen cabida la más variada gama de profesiones y estatus social.

\section{NATURALEZA, VECINDAD Y RESIDENCIA}

El análisis de los protocolos notariales gaditanos señala a la ciudad atlántica como un gran núcleo receptor de personas de muy diverso origen, atraídos por el auge comercial experimentado por la misma, sobre todo a raíz de su conversión en cabeza del monopolio del comercio con Indias; estos emigrados establecieron unos lazos con la ciudad que si bien en un alto porcentaje fueron meramente coyun- 
turales, en otros casos consolidaron un asentamiento definitivo. No obstante, el principal motivo de estancia en la ciudad para un gran número de personas será la utilización de su puerto para empreder el viaje a América, en este sentido podemos afirmar, a la luz de la documentación consultada, que Cádiz era una verdadera plataforma de emigración a Indias.

De la documentación inventariada hemos obtenido un total de 2.023 personas que utilizan el puerto de Cádiz para efectuar su viaje a América, el origen o naturaleza de estos emigrados queda especificado en el siguiente cuadro:

\begin{tabular}{lrr} 
Origen & $\mathbf{N .} \mathbf{.}^{-}$ & $\mathbf{\%}$ \\
\hline Galicia & 62 & $7,8 \%$ \\
P. Vasco y Navarra & 115 & $14,5 \%$ \\
Cantabria & 20 & $2,5 \%$ \\
Rioja & 20 & $2,5 \%$ \\
Asturias & 13 & $1,6 \%$ \\
Aragón & 5 & $0,6 \%$ \\
Castilla-León & 55 & $7,0 \%$ \\
Castilla-La Mancha & 17 & $2,1 \%$ \\
Cataluña & 21 & $2,6 \%$ \\
Levante & 15 & $1,8 \%$ \\
Baleares & 9 & $1,1 \%$ \\
Canarias & 4 & $0,5 \%$ \\
Extremadura & 3 & $0,4 \%$ \\
Andalucía Oriental & 24 & $3,0 \%$ \\
Andalucía Occidental & 286 & $36,0 \%$ \\
Indias & 26 & $3,2 \%$ \\
Extranjeros & 100 & $12,5 \%$ \\
& & \\
TOTAL & 795 & $100,0 \%$ \\
No especifican & 1.228 & $60,7 \%$
\end{tabular}

Lo primero que notamos en el cuadro anterior es el alto índice de individuos que silencia su procedencia, lo que repercute consecuentemente en la fiabilidad de la muestra, más aún si tenemos en cuenta que es el grupo de "residentes", es decir, aquellos que vienen a Cádiz por un corto período, el que cuenta con un mayor número de personas que omiten este dato. Y son ellos precisamente los que vienen a Cádiz con el único fin de concertar un pasaje que les lleve a 
América, los auténticos emigrantes. A pesar de las reservas que impone lo dicho, el cuadro ofrece datos muy interesantes para calibrar el aporte de cada una de las comunidades a la emigración durante estos años.

La fuerte presencia de andaluces en la emigración trasatlántica es una constante desde el siglo XVI. Ya Boyd-Bowman establecía para el período de 1540-59 un 35\% de emigrantes de origen andaluz frente a un 45\% procedente de Extremadura y las dos Castillas. En 1689 del millar de habitantes españoles en México, el 30\% eran andaluces, el $28 \%$ castellanos y el 2,9\% extremeños (13). Para este siglo según Lourdes Díaz-Trechuelo, el mayor aporte de emigrantes andaluces procede del Reino de Sevilla con un $78,39 \%$ y, dentro de él la capital con el $55,25 \%$ del total registrado (14). Vemos como en el siglo XVIII, y a partir de nuestros datos, la emigración andaluza sigue siendo la más importante, con un 39\% del total y dentro de ella es la zona occidental, fundamentalmente las provincias de Cádiz y Sevilla, las que aportan el mayor número de efectivos. Este predominio de Andalucía Occidental es consecuencia de su tradicional apertura al mar y de su condición de sede del monopolio con América; no es extraño por tanto que sea Cádiz la ciudad que aporte un mayor número de emigrados $(19,3 \%)$ (15). Las causas de la fuerte y constante emigración andaluza durante toda la Edad Moderna deben buscarse tanto en la especial situación del agro andaluz, con un aplastante predominio de la gran propiedad junto con sus secuelas: población concentrada, creación de una auténtica masa de proletariado agrícola y graves problemas sociales, como a su gran densidad humana. En años normales los jornaleros vivían al límite de la subsistencia o alcanzándola a duras penas gracias al trabajo de mujeres y niños, la rebusca, el pequeño hurto de frutos y la mendicidad (16). Para Olavide eran "los hombres más infe-

(13) MORNER, Magnus: op. cit., p. 62, Vid. Boyd-Bowman: Indice Geobiográfico de más de 56 mil pobladores de la América Hispánica, 5 vols., México, 1985.

(14) DIAZ-TRECHUELO, Lourdes: "La emigración familiar andaluza a América en el siglo XVII" en La emigración española... op. cit., pp. 195-196.

(15) Para MORALES PADRON, Francisco: "Sevilla y América", Atlántida, VIII, 1970, p. 626, "la emigración sevillana viene determinada por su condición de puerto único". La importancia de los factores de "atracción" con respecto a Andalucía fue señalada ya por RODRIGUEZ ARZUA, J.: "Las regiones españolas y la población de América, 1509-1538". Revista de Indias, año VIII, n.30, 1947, pp. 695-748.

(16) DOMINGUEZ ORTIZ, A.: Sociedad y Estado en el siglo XVIII español, Barcelona, 1976, pp. 213-218. 
lices que yo conozco en Europa... Estos hombres la mitad del año son jornaleros y la otra mitad mendigos" (17).

Que la presencia de emigrantes procedentes de la línea costera septentrional se intensificaba fuertemente durante el siglo XVIII, era algo que ya indicaba Magnus Morner en su citado artículo. Los datos obtenidos por nosotros confirman este supuesto, llegando a alcanzar el 29,5\% los emigrados procedentes del norte de España. De entre ellos, con un $14,5 \%$ destacan los vascos y navarros cuya presencia en América está constatada desde el comienzo mismo de la conquista. Sobresalen en la expansión hacia el norte del virreinato novohispano; la fundación y primeros establecimientos de las costas californianas van unidas a personajes como Cristóbal y Juan de Oñate, Juan de Tolosa, Diego y Francisco de Ibarra, Francisco de Urdiñola, Martín de Zabala y Sebastián de Vizcaíno. Asimismo, los primeros obispos de México (Zumárraga) y Oaxaca (Mendieta) encabezan una larga lista de notables eclesiásticos vascos en la Nueva España del XVI (18). Pero si bien según Boyd-Bowman los vascos presentes en México en el período 1560-79 sobrepasaban ligeramente el 1\% de los inmigrantes europeos, su presencia se había incrementado hasta el 14,5\% un siglo más tarde, en 1689. Su destacada presencia en la América española del siglo XVII es también sugerida por otras fuentes: durante la rivalidad que dividió a la población española en los campos mineros de Potosí en los años de 1620 y 1640 , uno de los bandos estaba compuesto por vascos que se opusieron a todos los demás, peninsulares y criollos (19). La conjunción de una alta densidad de población, basada en un elevado indice de fertilidad y escasez de tierra disponible, tanto por la configuración física del país como por las limitaciones impuestas por el sistema hereditario (único heredero), la tradición marítimo-comercial, la resistencia del campesino vasco al trabajo asalariado, etc., junto con otras causas de origen más coyuntural, como la pérdida de una buena

(17) Pablo de OLAVIDE: "Informe de Olavide sobre la ley agraria", ed. R. CARANDE, Boletin de la Real Academia de la Historia, CXXXIX, 1956, pp. 370-462. Este informe, uno de los más documentados sobre la situación del campo español y la situación en Andalucía ha sido estudiado también por ORTEGA LOPEZ, M.: "Los informes de los intendentes andaluces y el expediente de la ley agraria: una vía reformista en el campo español en la segunda mitad del siglo XVIII", Axerquia, 4, 1982, pp. 101-123.

(18) VAZQUEZ DE PRADA VALLEJO, V. y AMORES CARREDANO, J.B.: "La emigración de navarros y vascongados al Nuevo Mundo y su repercusión en las comunidades de origen" en La emigración española... op. cit., pp. 133-142.

(19) MORNER, Magnus: op. cit., pp. 20-21. 
parte de la actividad bacaladera a manos inglesas, durante el siglo XVIII, períodos de escasez y hambre, como los conocidos de 1690-95, 1708-18 ó 1846-47, explicarían en gran medida la importante presencia vasca en las tierras americanas (20).

Las primeras señales de emigración popular de gallegos a las Indias datan de comienzo del siglo XVIII (durante el siglo XVI y XVII fue básicamente intrapeninsular), constatándose ésta a través de las menciones de emigrantes muertos en América en la documentación notarial y judicial. Diversos estudios nos hablan de una intensificación de la emigración gallega a raíz de las crisis agrarias de la década de 1690, emigración vertida principalmente sobre Andalucía siendo Cádiz uno de los principales puntos de destino (21). No obstante que gran parte de esta emigración utilizaba Cádiz como puerto de embarque hacia América queda atestiguado por las cifras que ofrecemos donde el porcentaje de emigrantes gallegos roza el $8 \%$.

Con un porcentaje sensiblemente menor pero no por ello poco importante (son regiones uniprovinciales) aparecen Cantabria $(2,5 \%)$, Asturias $(1,6 \%)$ y La Rioja $(2,5 \%)$. Los factores de expulsión como en los casos anteriores son complejos. El espectacular crecimiento de la producción agraria tras la introducción del maíz y la roturación de

(20) VAZQUEZ DE PRADA VALLEJO, V. y AMORES CARREDANO, J.B.: op. cit., pp. 137-138. La pobreza y el mayorazgo son también, para PILDAIN SALAZAR, M. ${ }^{a}$ P., factores explicativos claves a la hora de entender la emigración vasca a América entre 1840-1870, aunque en este momento histórico las consecuencias de la primera guerra carlista ocupen un lugar predominante: Ir a América. La emigración vasca a América (Guipuzzcoa, 1840-1870), San Sebastián, 1984. El mayorazgo, en cambio, no parece constituir un factor clave en la emigración asturiana según BARREIRO MALCON, B.: "Ritmos, causas y consecuencias de la emigración asturiana a América, 1700-1900", en Emigración española y portuguesa a América, Actas del II Congreso de la Asociación de Demografia..., op. cit., pp. 73-88.

(21) Las causas de la emigración gallega fueron analizadas por MEIJIDE PARDO, A.: "La emigración gallega intrapeninsular en el siglo XVIII", Estudios de Historia Social de España, IV, 2, CSIC, Madrid, 1960, pp. 489-522. También EIRAS ROEL profundiza en las causas demográficas y económicas de la misma a partir del concepto de "superpoblación relativa": "En torno a la emigración gallega a América en el siglo XIX. Algunas consideraciones a la luz del ejemplo canario", Revista de la Facultad de Geografia e Historia, núm. 4, 1989, pp. 225-240.

Por lo que respecta al papel de Cádiz como punto de destino frecuente de muchos gallegos desde finales del XVII, ha sido destacado por GONZALEZ LOPO, D.: "Una aproximación a la emigración de la Galicia Occidental entre mediados del siglo XVII y el primer tercio del XX, a través de las fuentes de Protocolos y Archivos Parroquiales", Revista Da Comision Galega do Quinto Centenario, 6, Xunta de Galicia, 1991, pp. 141-142 y por EIRAS ROEL, A.: "La emigración gallega a América. Panorama General", en La emigración a Ultramar..., op. cit., p. 20. 
comunales fue insuficiente para corregir el déficit estructural de la agricultura cántabra. Hasta bien entrado el siglo XVIII, la producción fue incapaz de crecer permaneciendo estancada. En cuanto a Asturias, ya los propios contemporáneos oriundos de la región como Jovellanos o Campomanes mostraban su preocupación por el panorama socioeconómico que ofrecía, lo que obligaba a sus gentes a encontrar el sustento fuera del Principado. Ya entrado el siglo XVIII serían frecuentes las migraciones a América según nơs dice Jovellanos, de donde "volvían ricos metiéndose a terrazgueros" (22). Los datos obtenidos confirman el descenso de la aportación castellana a la emigración ultramarina (9\%). La falta de estudios sobre esta emigración durante el siglo XVII nos impide contemplar un cuadro evolutivo de su aportación mas sabemos que ésta fue muy importante durante el siglo XVI (23). Según nuestros datos, Burgos sería el principal centro emisor con un $50 \%$ del total de emigrados de Castilla-León. Por otra parte no deja de ser significativo el pequeño papel representado por Extremadura con sólo un $0,4 \%$; esta pérdida de peso de los extremeños en América está documentada ya para finales del XVII (en México sólo representaban un $2,9 \%$ del total de españoles en 1689) (24).

La escasa presencia catalana en América está confirmada para los siglos XVI y XVII (25), no obstante, las últimas aportaciones hablan de que si bien el comercio catalán no generó una emigración numerosa a América, no dejó de estar presente en el comercio americano. Para el siglo XVI se barajan porcentajes siempre inferiores al 1\%, mientras que en 1689 en la ciudad de México los catalanes representan el 5,4\% del total de españoles (26). Desde el segundo tercio del siglo XVIII, exis-

(22) JOVELlaNOS, G.M.: "Carta sexta a Ponz" en Obras, Madrid, 1956, p. 290. Vid. asimismo, OJEDA SAN MIGUEL, J.L.: Campesinos, emigrantes, indianos, emigración y economía en Asturias, Oviedo, 1985.

(23) MORNER, Magnus: op. cit., pp. 19-24.

(24) RUBIO MAÑE, J.L.: "Gente de España en la ciudad de México, año 1689", Boletín del Archivo General de la Nación, vol. III, 1966.

(25) Los trabajos de MARTINEZ SHAW: "Cataluña y el comercio con América. El fin de un Debate" en Boletín Americanista, 30, 1980, pp. 223-226. DELGADO RIBAS: "América y el comercio de Indias en la historiografía catalana (1892-1978)" en Boletin Americanista, 28, 1978, pp. 179-187; y VILAR, Pier: Catalunya dins l'Espanya mosderna, Barcelona, 1964, entre otros atribuyen esta mínima presencia catalana en los mercados americanos a las dificultades económicas del principado, abocado a la recuperación económica interior. YAÑEZ GALLARDO, César: "La emigración catalana a América. Una visión de largo plazo" en La emigración española... op. cit., pp. 171-178.

(26) RUBIO MAÑE, J.L.: op. cit., pp. 
ten evidencias de la presencia de naves catalanas en América. Según nuestros datos los catalanes sólo representan el 2,6\% del total de los viajeros a mediados del siglo XVIII lo que confirmaría la hipótesis que retarda, el primer auge del comercio y la emigración catalana hasta el último tercio del siglo XVIII (27). En este mismo artículo Delgado nos dice que en el monto total de la emigración catalana predomina un tipo social concreto; el pequeño comerciante o aprendiz de tal, que viaja como responsable -encomendero- de un cargo de mercancías con la misión de "beneficiar" en las colonias.

Un grupo numeroso de los embarcados a Indias son extranjeros $(12,5 \%)$; de ellos tres nacionalidades se reparten el $80 \%$ de sus efectivos: genoveses son el 35\%, franceses el $27 \%$ e irlandeses el $17 \%$. Sabido es que a los extranjeros les estaba prohibido asentarse y aun ni siquiera visitar las Indias, no obstante como muestra Richard Konetzke (28) existían mecanismos legales que matizaban esta prohibición; éstos eran fundamentalmente dos: el proceso de naturalización que tenía lugar en España antes de la marcha, y también desde finales del siglo XVI en adelante, la composición mediante el pago de tasas fijas en América. Esta situación no cambió con la llegada de los Borbones pues, no habiendo concluido aún la Guerra de Sucesión, Felipe $\mathrm{V}$ decretó una orden de expulsión contra los franceses establecidos en la isla de Santo Domingo. A lo largo del siglo se van sucediendo nuevas disposiciones restrictivas al comercio y emigración de los extranjeros en América. No es extraño si consideramos que durante la Guerra de Sucesión los extranjeros, debido a la incapacidad de abastecimiento desde España, monopolizaron prácticamente el mercado con las colonias. No obstante, el monopolio español tuvo sus limitaciones (las cifras que ofrecemos son prueba de ello) atribuibles en unas ocasiones a la corrupción reinante en los organismos oficiales y en otras a las escapatorias legales previstas dentro de los propios marcos del régimen prohibitivo, las licencias y las naturalizaciones (29).

(27) DELGADO RIBAS, J.M.: "La emigración española a América latina durante la época del comercio libre (1765-1820). El ejemplo catalán", Boletín Americanista, 32, 1987, pp. 115-137.

(28) KONETZKE, Richard: "Legislación sobre inmigración de extranjeros a América durante la época colonial". Revista Internacional de Sociología, julio-diciembre de 1945, n. ${ }^{\circ} 11-12$, p. 272.

(29) GARCIA-BAQUERO, Antonio: Cádiz y el Atlántico (1717-1778), Sevilla, 1976. 


\section{ESTADO CIVIL}

De los documentos consultados, testamentos, licencias, prorrogaciones y poderes, podemos extraer una serie de datos que nos ayudaran a perfilar un tipología básica de aquél emigrante que utilizó nuestra ciudad como puerto de embarque hacia su destino americano.

La forma en la que se nos muestra la situación civil de cada emigrado es variada, claramente expuesta en unos casos, en otros se halla implícita en una serie de disposiciones y cláusulas del documento. Es importante señalar que en este segundo caso es más fácil reconocer una persona casada que a una soltera por varios motivos: la necesidad de autorización del cónyuge y por tanto de licencia; en el testamento aparece siempre consignado su situación civil, además de otros datos como el nombre del esposo o esposa, dote, arras, hijos, etc. Los poderes son otorgados a veces de forma recíproca entre esposos, o también ocurre que el marido antes de partir otorga a su esposa un poder con un determinado fin.

Distinto es el caso de los solteros ya que en numerosísimos casos no mencionan su estado civil siendo por tanto, a falta del dato claro, problemática su inclusión en este grupo. Hemos preferido considerar solteros aquellos que lo expresan claramente en su testamento o poder, así como los que solicitan poder para casarse. A pesar de ello, el grupo de los que no especifican puede ser considerado, a todos los efectos, como un grupo de solteros dado que no aparece la indispensable licencia y, en el testamento o poder no mencionan la boda, dejando como herederos a sus padres o hermanos, señal inequívoca de carecer de descendientes.

\begin{tabular}{lrr} 
Casados & 630 & $31,14 \%$ \\
Viudos & 47 & $2,32 \%$ \\
Divorciados & 1 & $0,04 \%$ \\
Solteros & 295 & $14,60 \%$ \\
No especifican & 1.050 & $51,90 \%$ \\
\multicolumn{1}{c}{ TOTAL } & 2.023 & $100,00 \%$
\end{tabular}

Vemos en el cuadro cómo el grupo de los solteros (solteros más los que no especifican) es mayoritario con un $66,5 \%$ del total seguidos de los casados con poco más del 30\%. A pesar de la imposibilidad de conocer la edad de los emigrados, el hecho de la presencia mayoritaria de solteros nos informa de una población joven que confirma la hipótesis, convertida en lugar común, de que los emigrantes volunta- 
rios son generalmente más bien jóvenes. Jordi Nadal Oller (30) estima que un $15 \%$ de la población masculina tendría de 16 a 25 años en la España de 1760 por lo que sería "lógico" un alto porcentaje de emigrantes pertenecientes a ese segmento de edad.

\section{PROFESION}

Dada la variedad de oficios y profesiones que aparecen en los documentos nos hemos visto obligados a encuadrarlos en cinco grandes grupos, siendo uno de ellos el de los individuos que nada dicen al respecto, bien porque no lo tenían, hipótesis que parece más plausible, bien porque no lo consideraran necesario o tuviesen razones para ocultarlo. No estaría de más aventurar que fuesen en su mayoría de origen campesino dado que son muy escasos los documentos en los que aparece explicitada esta ocupación, lo que causa estrañeza considerando que, como sabemos, en el Antiguo Régimen era mayoritaria.

\begin{tabular}{lrr} 
Tripulación & 472 & $23,3 \%$ \\
Comerciantes & 128 & $6,3 \%$ \\
Cargos públicos & 52 & $2,5 \%$ \\
Otros & 168 & $5,6 \%$ \\
No especifican & 1.203 & $59,4 \%$ \\
\multicolumn{1}{c}{ TOTAL } & 2.023 & $100,0 \%$
\end{tabular}

Puede parecer sorprendente el elevado número de personas que pertenecen a la tripulación de los navíos, pero hemos de tener en cuenta que una de las características de la navegación a vela con sus servidumbres técnicas, climatológicas y temporales, es precisamente la hipertrofia de las tripulaciones (31). No realizaban un solo viaje en su vida, normalmente salían y volvían con la flota, si bien muchos de ellos una vez llegados al Nuevo Continente, abandonaban sus funciones y comenzaban una nueva vida en América. En otras ocasiones, la tripulación a la vez que desempeñaban su actividad en el barco ejercían actividades mercantiles bien por cuenta propia o ajena, llevando a su cargo algún que otro cajón con mercancías, poderes para cobrar, etc.

(30) NADAL OLLER, J.: La población española (siglos XVI-XX), Barcelona, 1966, pp. 76-78.

(31) GARCIA BAQUERO, A.: Cádiz y el Atlântico, op. cit., pp. 287-289. 
A pesar del modesto 6\% que declaran su condición de hombres del comercio, hemos de presumir que su número fuese mayor pues hemos tenido ocasión de comprobar que son muchos los comerciantes que no mencionan su profesión. A pesar de tener constancia de ello hemos preferido incluirlos en el grupo de los que no especifican.

El grupo "otros" está integrado por los religiosos (55 que representan el 2,7\%), que en numerosas ocasiones forman parte de los navíos como capellanes; militares $(55,2,7 \%)$; profesiones liberales (34, $1,7 \%$ ) donde abundan los cirujanos, abogados, escribanos, etc.; artesanos $(11,0,5 \%)$ y un pequeño grupo compuesto de mozos, criados, aprendices, etc. $(13,0,6 \%)$.

En resumen, nos encontramos ante un conjunto mayoritario de individuos que afrontan la emigración sin ningún tipo de cualificación profesional, representado por ese casi $60 \%$ que parece no tener una ocupación específica; un nutrido grupo de profesionales de la navegación (Aquí hemos incluido a los que añaden a su profesión el apelativo "de navío", tales como cirujanos, escribanos, barberos, carpinteros, contables, etc.), difícilmente cuantificables como emigrantes; una significativa proporción de comerciantes que todo apunta a que fuese mayor y una mínima representación de profesionales cualificados como artesanos, militares, profesiones liberales, etc.

\section{DESTINO}

Para simplificar la diversidad de puntos de destino hemos clasificado éstos en seis zonas de llegada donde se integrarían los distintos puertos y ciudades:

$\begin{array}{llr}\text { Nueva España } & 855 & 42,2 \% \\ \text { Tierra firme } & 321 & 15,8 \% \\ \text { B. Aires } & 255 & 12,6 \% \\ \text { Mardel Sur } & 237 & 11,7 \% \\ \text { Caribe } & 158 & 7,8 \% \\ \text { Indias } & 278 & 13,7 \%\end{array}$

Como vemos el destino de los emigrantes es variado cubriendo en gran parte la totalidad del subcontinente americano. Lógicamente el destino que aparece señalado en las escrituras es el del puerto o ciudad de arribada de las flotas, por lo que en la mayoría de los casos desconocemos el destino final, obteniendo de esta forma sólo una idea aproximativa de la distribución espacial de estos emigrantes en tierras americanas. 
Se observa como casi la mitad de ellos se dirigen al virreinato de Nueva España, cifra que, en gran parte, coincide con las que ofrece Díaz-Trechuelo como destino mayoritario de la emigración andaluza en el siglo XVII $(39,85 \%)$ (32). Nos encontramos además, con un grupo importante de emigrantes $(13,7 \%)$ que no especifican el lugar hacia el que se dirigen limitándose a señalar que viajan a América sin precisar más; esto nos hace pensar que nos hallamos ante un grupo de personas que buscan fortuna y un lugar para establecerse, que su marcha no obedece a un asunto o negocio determinado.

Por último hemos de precisar que la mayoría de los emigrantes que se dirigen a Nueva España lo hacen al puerto de Veracruz; mientras que los que viajan al Caribe lo hacen sobre todo al puerto de La Habana.

\section{OTROS DATOS SOCIOLOGICOS}

Podemos completar este perfil del emigrante con otros datos obtenidos en la muestra que si bien no son abundantes sí pueden ser significativos. Esto ocurre con el nivel de alfabetización manifestado en la firma del documento; así vemos como los firmados por hombres alcanzan el 92,9\% mientras que sólo firman el 36,3\% de las mujeres que otorgan documentos. En cuanto a las características de la firma algunas de ellas estaban bien realizadas, denotando cierta soltura con la pluma, mientras que en otras, usual en los documentos femeninos, se apreciaba una mera memorización de los signos o una escasa práctica de la escritura.

Un dato que nos interesó desde un principio fue la frecuencia de viaje de algunos de los individuos recopilados en la muestra. Nos llamó la atención que en un corto período de tiempo se repetían los datos de una misma persona. Pudimos comprobar cómo 122 personas realizaron el mismo viaje dos veces en los diez años de la muestra; mientras que fueron 21 los individuos que lo realizaron en más de dos ocasiones. Si bien la cifra es poco significativa comparada con el total de personas inventariadas (4\%), no deja de ser interesante comprobar cómo un viaje que entrañaba tantos riesgos, fuese realizado en más de una ocasión en una sola década de la vida de una persona. Como es fácil suponer en su mayoría eran miembros de la tripulación; en otros casos, también frecuentes, eran comerciantes.

(32) DIAZ-TRECHUELO, Lourdes: "La emigración familiar andaluza a América en el siglo XVII", La emigración ... op. cit., p. 197. 
Si contrastamos la documentación obtenida en el Archivo General de Indias con la obtenida en el Archivo de Protocolos de Cádiz vemos que el total de personas que aparecen en este último archivo (2.023) representan el 58,2\% del total de los que viajaron en esa década de forma legal (3.470), es decir, mediante la correspondiente licencia de embarque. Como podemos ver el peso de la muestra gaditana es considerable. Si comparamos los datos de viajeros según el sexo vemos que el porcentaje de mujeres que aparecen en la muestra sevillana es mayor:

SEVILLA

CADIZ

\begin{tabular}{lclr}
\hline TOTAL & 3.470 & TOTAL & 2.023 \\
Hombres & $3.251(93,6 \%)$ & Hombres & $2.012(99,5 \%)$ \\
Mujeres & $219(6,3 \%)$ & Mujeres & $11(0,5 \%)$
\end{tabular}

La diferencia porcentual viene determinada por la diferencia de los documentos tratados, así mientras que en el documento gaditano no se contempla la figura del acompañante (el protagonismo jurídico de la mujer en el Antiguo Régimen está relegado a un segundo plano), éste sí aparece en la licencia de embarque del Archivo de Indias. Un ejemplo ilustrativo puede ser el del marqués 'de las Amarillas; mientras que en Sevilla aparece con dos acompañantes femeninas en la muestra de Cádiz sólo aparece una mujer acompañándole como criada. Naturalmente al ser tan poco profuso el número de mujeres que viajaban los porcentajes varían considerablemente con unos cuantos ejemplos semejantes.

Por último hemos de señalar los casos de defunción constatados en la documentación notarial tanto los referidos a fallecimientos durante el viaje, considerando los peligros de una navegación de tales características, como los acaecidos en el lugar de destino. Así hemos encontrado referencias a 95 fallecidos mediante documentos otorgados por mujeres, bien solicitando ayuda para buscar a su marido, o bien solicitando la parte correspondiente en los bienes de su cónyuge. Uno de estos casos puede ser el de una mujer muerta en Indias por causas naturales y gracias al documento otorgado por una sobrina reclamando su parte correspondiente en la herencia pudimos detectarlo.

\section{CONCIUSIONES}

Con los datos obtenidos podemos construir una ficha tipo del emigrante que usó Cádiz como puerto de embarque para su viaje a las Indias durante esta década: 


\begin{tabular}{|c|c|}
\hline Sexo & Varón \\
\hline Estado civil & Soltero/recién casado \\
\hline Edad & Entre $25-40$ años \\
\hline Procedencia & Andalucía Occidental \\
\hline Profesión & Sin oficio \\
\hline Motivo del viaje & Buscarse la vida \\
\hline Duración & Sin limitación de tiempo \\
\hline Destino & Nueva España \\
\hline Nivel Social & Medio/Bajo \\
\hline Documento otorgado $. . . \ldots \ldots \ldots \ldots \ldots . . . . .$. & Poder/Testamento \\
\hline
\end{tabular}

No queremos finalizar sin volver a recordar la validez del documento notarial como fuente para el estudio de la emigración ultramarina. Validez que creemos queda patente en los datos obtenidos en el presente trabajo, aunque con ello evidentemente no creemos haber agotado todas las posibilidades que nos brinda este tipo documental. Así, a través de ella, constatamos la presencia de individuos que hacen el viaje sin haber solicitado la reglamentaria licencia de embarque con lo que podríamos detectar de algún modo la emigración clandestina. Aspectos como las relaciones entre los individuos que han marchado y los que han quedado en la metrópolis, el destino final de muchos de ellos, así como sus éxitos o fracasos, etc., puntos que evidentemente desbordaban los límites que nos habíamos impuesto para el presente estudio, pueden salir a la luz y aportar datos que consigan aclarar determinados problemas que aún hoy pesan sobre este, por otra parte tan sugestivo, tema histórico.

INDICE DE LEGAJOS CONSULTADOS EN EL ARCHIVO GENERAL DE INDIAS DE SEVILLA

SECCION ULTRAMAR:

Informaciones y Licencias de pasajeros.

\begin{tabular}{ll} 
Año & N.o de Orden \\
\hline 1750 & 5.491 \\
1751 & $5.492 / 93$ \\
1752 & 5.494 \\
1753 & 5.495 \\
1754 & 5.496 \\
1755 & 5.497
\end{tabular}




\begin{tabular}{ll} 
Año & N.o de Orden \\
\hline 1756 & $5.498 / 99$ \\
1757 & 5.500 \\
1758 & 5.501 \\
1759 & 5.502 \\
1760 & $5.503 / 04$
\end{tabular}

INDICE DE LEGAJOS CONSULTADOS EN EL ARCHIVO HISTORICO PROVINCIAL DE CADIZ

\begin{tabular}{llrr} 
AÑo & NOMBRE & N.o NOTARIA & N.o ORDEN \\
\hline \multirow{2}{*}{ Als0 } & 3 & 766 \\
Alcalá Guerrero, Nicolás & 13 & 2.549 \\
Barra Mendiguren, Simón & 10 & 1.842 \\
Camacho, José Antonio & 15 & 3.627 \\
Cárrega, Juan & 9 & 1.610 \\
Castellanos, Francisco & 11 & 2.173 \\
Ibídem & 31 & 5.934 \\
Costa Tello, Juan & 6 & 1.170 \\
Delgado Borjas, Miguel V. & 7 & 1.316 \\
Fuenseca y Cabrera, Felipe & 18 & 4.256 \\
González, Manuel Hilario & 1 & 31 \\
Hinojosa, José de & 24 & 5.543 \\
Huertas, Francisco de & 14 & 3.132 \\
Macharelo, Juan & 8 & 1.457 \\
Mateo Guerrero, Gerónimo & 16 & 3.777 \\
Montes, Juan Antonio & 20 & 4.959 \\
Pacheco Guzmán, Francisco & 23 & 5.339 \\
Pérez Angulo, Francisco & 21 & 5.088 \\
Pisson, Lorenzo & 5 & 1.026 \\
Pisson, Lorenzo & 17 & 3.837 \\
Rodríguez, Matías & 19 & $4.480 / 81$ \\
Ruiz Yagües, Fernando & 2 & 377 \\
Salgado, Juan Antonio & 25 & 5.745 \\
San Martín, José de & 4 & 885 \\
Vaamonde, José & 12 & $2.441 / 42$ \\
& & \\
Alcalá Guerrero, Nicolás & 3 & 767 \\
Arana Mendiguren, Simón & 13 & 2.549 \\
1751 & &
\end{tabular}


Aransáenz, Juan de 22

Barreda, Diego de la

5.164

Camacho, José Antonio

1.843

Cárrega, Juan

3.628

Castellanos, Francisco

1.610

Castellanos, Francisco

Costa Tello, Juan

10

15

2.174

Delgado Borjas, Miguel V.

5.934

31

1.170

Ibídem

886

González, Manuel Hilario

1.317

Herrera Vergara, Manuel

Hinojosa, José de

$4.257 / 58$

Huertas, Francisco de

5.543

Macharelo, Juan

3.133

Mateo Guerrero, Gerónimo

1.458

Montes, Juan Antonio de

3.777

Pacheco Guzmán, Francisco

4.960

Pérez Angulo, Francisco

5.340

Pisson, Lorenzo

5.089

Pisson, Lorenzo

1.027

Rodríguez, Matías

3.837

Ruiz Yagües, Fernando

4.482

Salgado, Juan Antonio

378

Vaamonde, José

5.746

25

12

$2.443 / 44$

1752 Alcalá Guerrero, Nicolás

768

Arana Mendiguren, Simón

2.550

Aransáenz, Juan de

5.165

Barreda, Diego de la

1.844

Cárrega, Juan

1.611

Castellanos, Francisco

2.175

Castellanos, Francisco

5.934

Costa Tello, Juan

1.171

Delgado Borjas, Miguel V.

1.318

González, Manuel Hilario

Herrera Vergara, Manuel

$4.257 / 58$

Hinojosa, José de

5.543

Huertas, Francisco de

3.134

López de Castro, Juan P. 
Macharelo, Juan

Mateo Guerrero, Gerónimo

Mateos, Juan Vicente

3.778

Montes, Juan Antonio de

3.629

Pacheco Guzmán, Francisco

4.961

Pérez Angulo, Francisco

16

15

5.341

Pérez, Tomás

5.090

Pisson, Lorenzo

887

Rodríguez, Matías

Ruiz Yagües, Fernando

20

23

21

Salgado, Juan Antonio

$4.485 / 86$

378

Soldevilla, Francisco Javier

5.745

Vaamonde, José

3.629

15

2.445

1753 Alcalá Guerrero, Nicolás

768

Arana Mendiguren, Simón

2.550

Aransáenz, Juan de

5.165

Barrera, Diego de la

1.845

Calle, Bernardo de la

1.171

Cárrega, Juan

1.612

Castellanos, Francisco

2.176

Castellanos, Francisco

5.934

Delgado Borjas, Miguel V.

1.318

González, Manuel Hilario

Herrera Vergara, Manuel

Huertas, Francisco de

3.134

López de Castro, Juan P.

3.838

Macharelo, Juan

1.460

Mateo Guerrero, Gerónimo

3.778

Mateos, Juan Vicente

3.630

Montes, Juan Antonio de

4.962

Pacheco Guzmán, Francisco

5.096

Pisson, Lorenzo

$1.030 / 31$

Rodríguez, Matías

Ruiz Yagüe, Fernando

Salgado, Juan Antonio

Soldevilla, Francisco Javier

Vaamonde, José 
1754 Alcalá Guerrero, Nicolás

Arana Mendiguren, Simón

2.550

Barreda, Diego de la

1.846

Calle, Bernardo de la

1.171

Cano, Diego

3.778

Cárrega, Juan

1.613

Castellanos, Francisco

2.177

Castellanos, Francisco

5.934

Delgado Borjas, Miguel V.

González, Manuel Hilario

Herrera Vergara, Manuel

Huertas, Francisco de

López de Castro, Juan P.

3.839

Macharelo, Juan

1.461

Mateo, Juan V.

3.631

Montes, Juan Antonio de

4.963

Pacheco Guzmán, Francisco

5.343

Pisson, Lorenzo

1.032

Pérez Angulo, Felipe

1.614

Rodríguez de Guerra, Luis

Rodríguez, Matías $4.489 / 90$

Salgado, Juan Antonio

Soldevilla, Francisco J.

Vaamonde, José

2.448

1755 Alcalá Guerrero, Nicolás

Arana Mendiguren, Simón

Barrera, Diego de la

Calle, Bernardo de la

1.172

Cano, Diego

3.779

Cárrega, Juan

Castellanos, Francisco

2.178

Castellanos, Francisco

5.934

Delgado Borjas, Miguel V.

González, Manuel Hilario

Herrera Vergara, Manuel

Hinojosa, José de

Huertas, Francisco de

López de Castro, Juan P. 
Macharelo, Juan

Mateos, Juan Vicente

Montes, Juan Antonio de

Pacheco Guzmán, Francisco

Pérez Angulo, Felipe

Pisson, Lorenzo

Rodríguez de Guerra, Luis

Rodríguez, Matías

Salgado, Juan Antonio

Soldevilla, Francisco J.

Vaamonde, José

1756 Alcalá Guerrero, Nicolás

Aransáenz, Juan de

Barreda, Diego de la

Calle, Bernardo de la

Cano, Diego

Cárrega, Juan

Castellanos, Francisco

Castellanos, Francisco

Delgado Borjas, Miguel V.

Estapar, Juan José

Herrera Vergara, Manuel

Hinojosa, José de

Huertas, Francisco de

Macharelo, Juan

Mateos, Juan Vicente

Montes, Juan Antonio de

Pacheco Guzmán, Francisco

Pérez Angulo, Francisco

Pisson, Lorenzo

Rodríguez de Guerra, Luis

Rodríguez, Matías

Salgado, Juan Antonio

Soldevilla, Francisco J.

Tirado, Ceferino Ignacio

Vaamonde, José

1757 Alcalá Guerrero, Nicolás

Aransáenz, Juan de
15

20

23

9

5

2

19

25

4

12

3

22

10

6

16

9

11

31

1

7

18

24

14

8

15

20

23

21.

5

2

19

25

4

17

12

3

22
1.462

3.632

4.964

5.344

1.614

1.033

379

$4.491 / 92$

5.750

889

2.449

772

5.166

1.849

1.172

3.779

1.616

2.179

5.934

32

1.320

$4.258 / 59$

5.544

3.136

1.463

3.633

4.965

5.345

5.091

1.034

5.751

$4.493 / 94$

5.751

890

3.839

$2.450 / 51$

772

5.166 


\begin{tabular}{|c|c|c|c|}
\hline AÑO & NOMBRE & N.o NOTARIA & 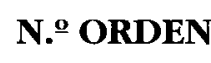 \\
\hline & Barreda, Diego de la & 10 & 1.849 \\
\hline & Calle, Bernardo de la & 6 & 1.172 \\
\hline & Cano, Diego & 16 & 3.779 \\
\hline & Cárrega, Juan & 9 & 1.617 \\
\hline & Castellanos, Francisco & 11 & 2.180 \\
\hline & Castellanos, Francisco & 31 & 5.934 \\
\hline & Cazorla, José & 4 & 891 \\
\hline & Delgado Borjas, Miguel V. & 1 & 32 \\
\hline & Estapar, Juan José & 7 & 1.321 \\
\hline & Fernández Suárez, Manuel & 12 & 2.452 \\
\hline & Herrera Vergara, Manuel & 18 & $4.258 / 59$ \\
\hline & Hinojosa, José de & 24 & 5.544 \\
\hline & Huertas, Francisco de & 14 & 3.136 \\
\hline & Macharelo, Juan & 8 & 1.464 \\
\hline & Martínez Temprado, Francisco & 13 & $2.551 / 52$ \\
\hline & Mateos, Juan Vicente & 15 & 3.634 \\
\hline & Montes, Juan Antonio de & 20 & 4.966 \\
\hline & Pacheco Guzmán, Francisco & 21 & 5.091 \\
\hline & Pisson, Lorenzo & 5 & 1.035 \\
\hline & Rodríguez de Guerra, Luis & 2 & 380 \\
\hline & Rodríguez, Matías & 19 & 4.495 \\
\hline & Salgado, Juan Antonio & 25 & 5.752 \\
\hline & Tirado, Ceferino Ignacio & 17 & 3.839 \\
\hline \multirow[t]{14}{*}{1758} & Alcalá Guerrero, Nicolás & 3 & 773 \\
\hline & Aransáenz, Juan de & 22 & 5.167 \\
\hline & Barreda, Diego de la & 10 & 1.850 \\
\hline & Calle, Bernardo de la & 6 & 1.173 \\
\hline & Cano, Diego & 16 & 3.780 \\
\hline & Cárrega, Juan & 9 & 1.618 \\
\hline & Castellanos, Francisco & 11 & 2.181 \\
\hline & Castellanos, Francisco & 31 & 5.934 \\
\hline & Cazorla, José & 4 & 891 \\
\hline & Cazorla, José & 13 & 2.552 \\
\hline & Estapar, Juan José & 7 & 1.322 \\
\hline & Fernández Pintado, Blas & 12 & 2.453 \\
\hline & Herrera Vergara, Manuel & 18 & $4.258 / 59$ \\
\hline & Hinojosa, José de & 24 & 5.544 \\
\hline
\end{tabular}


Huertas, Francisco de

Macharelo, Juan 14

Mateos, Juan Vicente

Montes, Juan Antonio de

1.465

Pacheco Guzmán, Francisco

15

3.635

Pérez Angulo, Francisco

20

4.967

Pisson, Lorenzo

Rodríguez de Guerra, Luis

23

5.347

21

5.092

Rodríguez, Matías

1.036

Salgado, Juan Antonio

5

2

380

Tirado, Ceferino Ignacio

1759 Alcalá Guerrero, Nicolás

Aransáenz, Juan de

Barreda, Diego de la

Calle, Bernardo de la

1.851

Cano, Diego

1.173

Cárrega, Juan

3.780

Castellanos, Francisco

1.619

Castellanos, Francisco

2.182

Cazorla, José

5.934

Estapar, Juan José

2.552

13

1.323

Fernández Pintado, Blas

381

Herrera Vergara, Manuel

Hinojosa, José de

Huertas, Francisco de

5.544

Mateos, Juan Vicente

3.137

14

15

3.636

Montes, Pedro Felipe de

20

4.968

Pacheco Guzmán, Francisco

Parra, Fernando de la

Pérez Angulo, Francisco

5.092

Pisson, Lorenzo

1.037

Rodríguez, Matías

4.497

Romero, Diego

892

Salgado, Juan Antonio

5.754

Tirado, Ceferino Ignacio

Zambrano, Juan 


\begin{tabular}{|c|c|c|c|}
\hline AÑNO & NOMBRE & 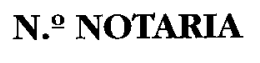 & N. ${ }^{\circ}$ ORDEN \\
\hline \multirow[t]{27}{*}{1760} & Alcalá Guerrero, Nicolás & 3 & 775 \\
\hline & Aransáenz, Juan de & 22 & 5.168 \\
\hline & Barreda, Diego de la & 10 & 1.852 \\
\hline & Calle, Bernardo de la & 6 & 1.174 \\
\hline & Cano, Diego & 16 & 3.781 \\
\hline & Cárrega, Juan & 9 & $1.620 / 21$ \\
\hline & Castellanos, Francisco & 11 & 2.183 \\
\hline & Castellanos, Francisco & 12 & 2.455 \\
\hline & Castellanos, Francisco & 31 & 5.934 \\
\hline & Cazorla, José & 13 & 2.553 \\
\hline & Estapar, Juan José & 7 & 1.324 \\
\hline & Fernández Pintado, Blas & 2 & 381 \\
\hline & Huertas, Francisco de & 14 & 3.137 \\
\hline & Mateos, Juan Vicente & 15 & 3.637 \\
\hline & Montes, Pedro Felipe de & 13 & $4.258 / 60$ \\
\hline & Montes, Pedro Felipe de & 20 & 4.969 \\
\hline & Pacheco Guzmán, Francisco & 23 & 5.349 \\
\hline & Parra, Fernando de la & 8 & 1.467 \\
\hline & Pérez Angulo, Francisco & 21 & 5.093 \\
\hline & Pisson, Lorenzo & 5 & 1.038 \\
\hline & Rodríguez, Matías & 19 & 4.498 \\
\hline & Román, Juan & 1 & 32 \\
\hline & Romero, Diego & 4 & 893 \\
\hline & Salgado, Juan Antonio & 25 & 5.755 \\
\hline & Tirado, Ceferino Ignacio & 17 & 3.840 \\
\hline & Torres, Antonio de & 24 & 5.545 \\
\hline & Zambrano, Juan & 12 & 2.455 \\
\hline
\end{tabular}

